\title{
Morphology transition mechanism from icosahedral to decahedral phase during growth of $\mathrm{Cu}$ nanoclusters
}

\author{
Alexey Tal, Peter Münger and Igor Abrikosov
}

\section{Linköping University Post Print}

\section{Tweet}

N.B.: When citing this work, cite the original article.

Original Publication:

Alexey Tal, Peter Münger and Igor Abrikosov, Morphology transition mechanism from icosahedral to decahedral phase during growth of $\mathrm{Cu}$ nanoclusters, 2015, Physical Review B. Condensed Matter and Materials Physics, (92), 2, 020102.

http://dx.doi.org/10.1103/PhysRevB.92.020102

Copyright: American Physical Society http://www.aps.org/

Postprint available at: Linköping University Electronic Press

http://urn.kb.se/resolve?urn=urn:nbn:se:liu:diva-120270 


\title{
Morphology transition mechanism from icosahedral to decahedral phase during growth of $\mathrm{Cu}$ nanoclusters
}

\author{
Alexey A. Tal, ${ }^{1,2, *}$ E. Peter Münger, ${ }^{1}$ and Igor A. Abrikosov ${ }^{1,2}$ \\ ${ }^{1}$ Department of Physics, Chemistry and Biology (IFM), Linköping University, SE-581 83, Linköping, Sweden \\ ${ }^{2}$ Materials Modeling and Development Laboratory, National University of Science and Technology “MISIS," 119049 Moscow, Russia
}

(Received 13 March 2015; revised manuscript received 11 May 2015; published 7 July 2015)

\begin{abstract}
The morphology transition from the thermodynamically favorable to the unfavorable phase during growth of freestanding copper nanoclusters is studied by molecular dynamics simulations. We give a detailed description of the kinetics and thermodynamics of the process. A universal mechanism of a solid-solid transition, from icosahedral to decahedral morphology in the nanoclusters, is proposed. We show that a formation of distorted $\mathrm{NC}$ during the growth process with islands of incoming atoms localized in certain parts of the grown particle may shift the energy balance between Ih and Dh phases in favor of the latter leading to the morphology transition deep within the thermodynamic stability field of the former. The role of diffusion in the morphology transition is revealed. In particular, it is shown that fast diffusion should suppress the morphology transition and favor homogeneous growth of the nanoclusters.
\end{abstract}

DOI: 10.1103/PhysRevB.92.020102

PACS number(s): 81.10.Aj, 61.46.Df, 81.10.Jt

The study of nanocluster (NC) growth has recently been the focus of many intense research activities [1], because of their unique properties and also the fundamentally new physical effects that occur in finite-sized systems [2]. NCs have found broad applications in catalysis [3-6], plasmonics [7], and biomedicine or photovoltaics [8-10]. All the applications require precise control of the $\mathrm{NC}$ growth process and an understanding of their properties. One of the most significant properties of the NCs is their morphology. It was shown that the favorable structure for copper nanocluster for $N<1000$ atoms is the Mackay icosahedron (Ih) [11,12], followed by the Marks decahedron (Dh) [13], which corresponds to the minimum energy structure for $1000<N<30000$ atoms in the NC. At larger $N$ the clusters optimal structure is fcc [14]. However, experiments demonstrate a significant amount of energetically unfavorable morphologies for different synthesis methods $[15,16]$. It is commonly accepted that it happens due to kinetics of the growth process. To study the growth kinetics is thus of fundamental importance. Baletto et al. [17] have demonstrated a possibility of the morphology transition in molecular dynamics (MD) simulations of silver nanoclusters and explained its mechanism [18]. They claimed that the transition from icosahedral (Ih) to decahedral (Dh) morphology goes through a melting of the cluster and a formation of the new morphology from the melted cluster. Lan et al. [19] proposed a qualitatively different mechanism for the transformation from $\mathrm{Dh}$ to Ih, where morphology goes through a solid-solid transition without the formation of an amorphous phase. On the other hand, it is known that the smaller the cluster size the more favorable the Ih phase should be. This means that in a real growth process the transition should go from Ih to Dh and it is very unlikely to go backwards. Thus it is much more relevant to study the Ih-Dh transition.

In this Rapid Communication, we report the mechanism of the solid-solid morphology transition that we have discovered in copper nanoclusters. Also, we provide a detailed explanation

\footnotetext{
*aleta@ifm.liu.se
}

of the conditions that induce the morphology transition, or on the contrary preserve the layer-by-layer growth. Also, the decisive role of diffusion in the morphology transition is exhaustively explained.

Note that the main interest in MD simulations of NCs addressed low-energy nanocluster growth techniques. However, recent developments of new NC synthesis methods, e.g., employing pulsed highly ionized plasma [20,21], have drawn attention to higher energies during the growth. The use of a plasma environment has several important advantages such as an increased growth rate and a wide choice of possible materials to grow. It has already been shown that the energy of the growth process significantly affects the kinetics of the growth process [22], a fact that should be accounted for in simulations. For instance, it was shown that the Coulomb interaction between the $\mathrm{NC}$ and impinging ions during the growth process will influence the angular distribution of the velocities of the impinging atoms. Consequently the angular distribution will affect the diffusion and local heating of the cluster surface.

We used the embedded-atom method potential with Foiles parametrization [23] to simulate the growth of copper NCs. We start our growth simulations from a seed. Based on the Baletto's analysis of structures and magic numbers for clusters [14], an icosahedral seed consisting of 147 atoms was chosen as our growth seed. Our choice covers the most relevant range of sizes for copper, since it includes the range where the morphology is set and the transition above the considered sizes is highly unlikely due to transition barrier height. Transitions in the clusters of the smaller size are less crucial for the final structure.

In the simulations new atoms were introduced around the cluster slightly outside the cutoff radius of the potential while the center of the cluster was kept fixed. Every 100 ps a new atom was introduced above the surface of the cluster with the speed and incidence representing one of the three cases: (IGA) thermal growth with speed of $0.03 \mathrm{eV}$ and angle of incidence varying from $0^{\circ}$ to $90^{\circ}$, (NI) plasma growth with speed of $1 \mathrm{eV}$ and zero incidence angle, and (FD) plasma growth with 
TABLE I. The fraction of the clusters that suffered a morphology transition for different temperatures. Normal incidence (NI) correspond to particles grown from atoms impinging on the cluster with normal incidence and a kinetic energy of $1 \mathrm{eV}$. Full distribution (FD) corresponds to particles grown from atoms with kinetic energy of $1 \mathrm{eV}$ and a distribution of incidences from normal to grazing. Particles grown from atoms with a kinetic energy of $0.03 \mathrm{eV}$ represent the IGA process.

\begin{tabular}{lccc}
\hline \hline Temp. (K) & NI (\%) & FD (\%) & IGA (\%) \\
\hline 400 & 28 & 16 & 26 \\
450 & 20 & 16 & 22 \\
500 & 10 & 10 & 18 \\
600 & 8 & 8 & 12 \\
700 & 4 & 2 & 2 \\
\hline \hline
\end{tabular}

speed of $1 \mathrm{eV}$ and angle of incidence varying from $0^{\circ}$ to $90^{\circ}$. Though the growth rates in our simulations are much higher than the experimental ones, we employ a thermostat to cool the cluster down to the correct temperature before a new particle impinge, this provides the correct conditions for the growth. The final structure was analyzed after 300 atoms were added to the seed. The temperature of the nanocluster was controlled by a Nosé-Hoover thermostat [24] and the speed of the impinging atoms corresponded to either the temperature of the cluster or a kinetic energy of $1 \mathrm{eV}$. These two cases were chosen to represent the inert-gas-aggregation (IGA) growth process or the conditions of the growth in a plasma, respectively [20].

We performed simulations in LAMMPS [25] for different temperatures, incident velocities, and angular distributions of the impinging atoms [22]. Every considered temperature, velocity, and angular distribution of the impinging atoms were studied in a series of 50 simulations. Analysis of the results showed significant fractions (up to $30 \%$ ) of nonicosahedral clusters among the final clusters. It is important to notice that every transition occurred at a different size due to the stochastic nature of the growth process. All results are assembled in Table I. Normal incidence and full distribution correspond to particles grown from atoms with an energy of $1 \mathrm{eV}$, simulating the NC growth in a plasma. We have considered two models of the growth, with atoms impinging on the surface with normal incidence and with the angular distribution derived in Ref. [22]. Results denoted as IGA correspond to a respective process simulated with the assumption that the impinging particles have a thermal energy $(0.03 \mathrm{eV})$. Note that, as was mentioned earlier, the thermodynamically favorable morphology for clusters of this size is icosahedral. Thus the kinetics of the growth process induces the morphology transition. However, the majority of the final clusters are still icosahedral, which raises the question: What is the difference between these two outcomes?

To address this question, we analyze in detail one typical simulation, where the structure changes from icosahedral to decahedral during the growth. Figure 1 shows coordination of atoms calculated with bond-angle analysis (BAA) [26]. The method was developed by Ackland and Jones [27] to distinguish fcc, hcp, and bcc coordination structures. From the bond vectors of the central atom the histogram of the

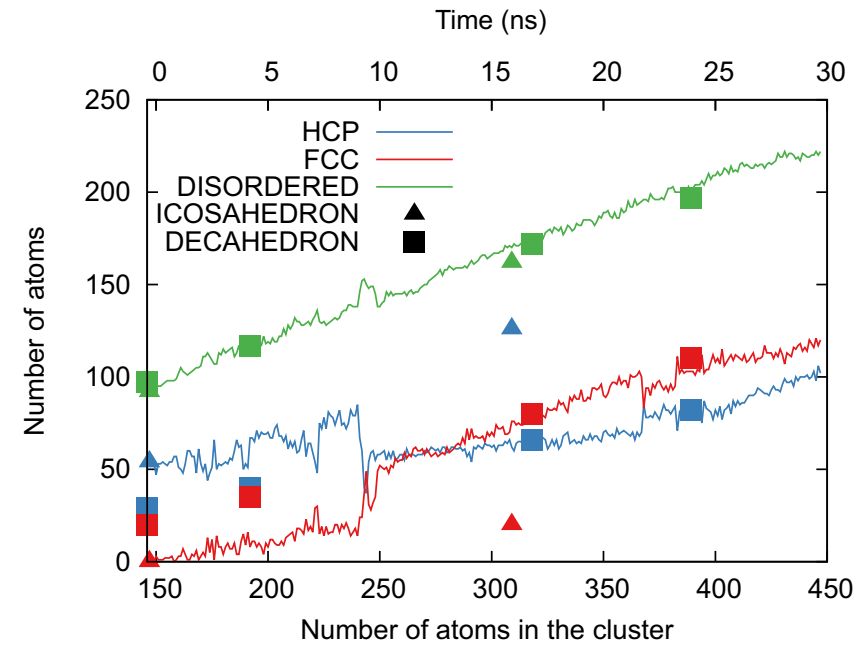

FIG. 1. (Color online) Bond-angle analysis of the cluster structure. The figure shows how the number of hcp, fcc, and disordered atoms changes with time, or equivalently the cluster size, during the growth process. Squares and triangles correspond to decahedrons and icosahedrons with magic number of atoms. Sizes: Ih $(147,309)$ and Dh $(146,192,318,389)$.

bond-angle cosines is computed and then used to determine the structure type by the heuristic decision rules. Initially the cluster consists of 147 atoms arranged in a perfect icosahedron with all atoms hcp coordinated. However, all surface atoms are identified as disordered by the BAA method. Thus we see that $\approx 50$ interior atoms are hcp coordinated and $\approx 100$ surface atoms are disordered. At the size of $\approx 250$ atoms the cluster undergoes the morphology transition from icosahedral to decahedral. At that point we see how the number of hcp-coordinated atoms drops down and the number of fcc-coordinated atoms abruptly increases. It clearly shows that part of the hcp-coordinated atoms change their local structure to fcc coordination, since we do not see any significant change in disordered atoms. After the transition fcc-coordinated atoms dominate over the hcp-coordinated ones for the remainder of the growth simulation. This means that there were no more morphology transitions. In Fig. 1, squares and triangles correspond to perfect decahedral and icosahedral clusters with magic numbers of atoms. Initially our structure is identical to Ih(147) and differs significantly from Dh(146). After the transition our cluster and $\mathrm{Ih}(309)$ have very different coordination of atoms, while it perfectly coincides with $\mathrm{Dh}(318)$ as well as $\mathrm{Dh}(389)$, which clearly confirms the transition.

In order to understand what induces the transition we analyzed the potential energy of the cluster as a function of time. Figure 2 shows the typical case described in the proceeding paragraph where the morphology transition takes place between incident atom 101 and 102, i.e., when the cluster has 248 atoms. We see that from 20 till 35 ps, after atom 101 was introduced, the potential energy of the cluster fluctuates. This means that the structure is far from perfect and that atoms on the surface change their positions and thereby change the potential energy of the system. However, after the transition, the structure falls into a potential well, where it minimizes 


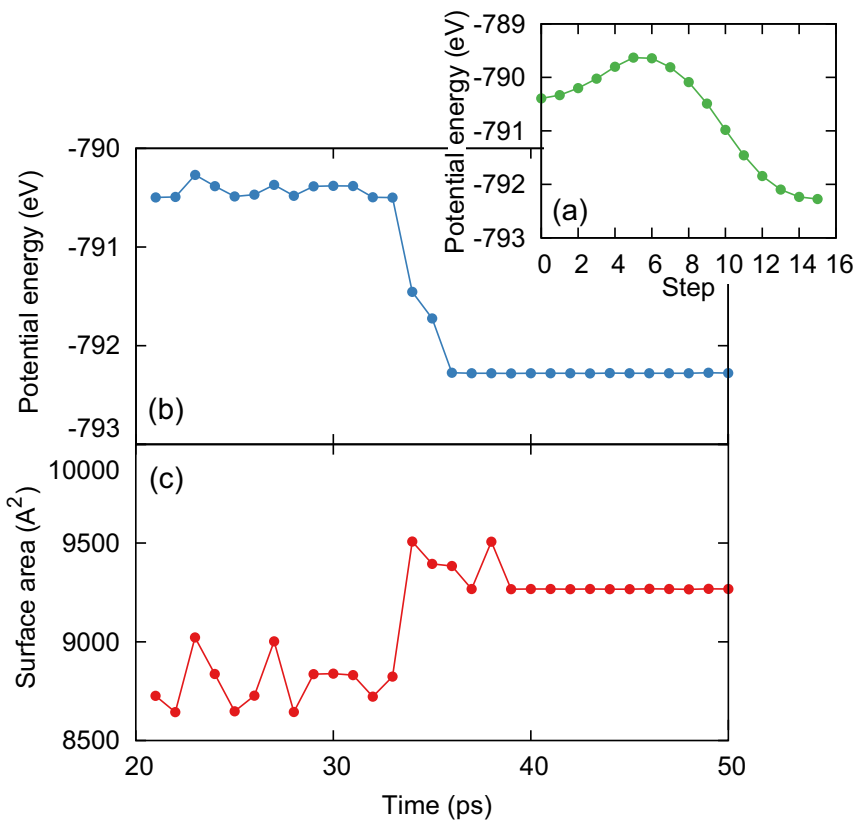

FIG. 2. (Color online) Data from the same simulation as in Fig. 1 but only for a short time period when the cluster consists of 248 atoms. (a) NEB analysis of the transition from the Ih to the Dh phase; (b) potential energy of the cluster as a function of time; and (c) dependence of the cluster surface area on the simulation time.

the potential energy and thus we see no more fluctuations of the energy. It is worth mentioning that the full transition takes approximately 2 ps and the whole time range of Fig. 2 corresponds to one point in Fig. 1. The energy between these two states is $1.8 \mathrm{eV}$. Also one can see in Fig. 2 that the surface area of the cluster changes significantly at the transition. The fluctuations of the potential energy correspond to changes of the surface area until the clusters undergo the morphology transition. Then the surface area promptly increases while fluctuations vanish. Before the transition all facets are very close to 111 and the surface of the nanocluster consists of 138 atoms. After the transition 100 facets appear and consist of 79 atoms, while 111 facets are formed by 59 atoms. In Fig. 3 one can see that the facets are not perfect and a certain amount of disordered atoms are present. In the case

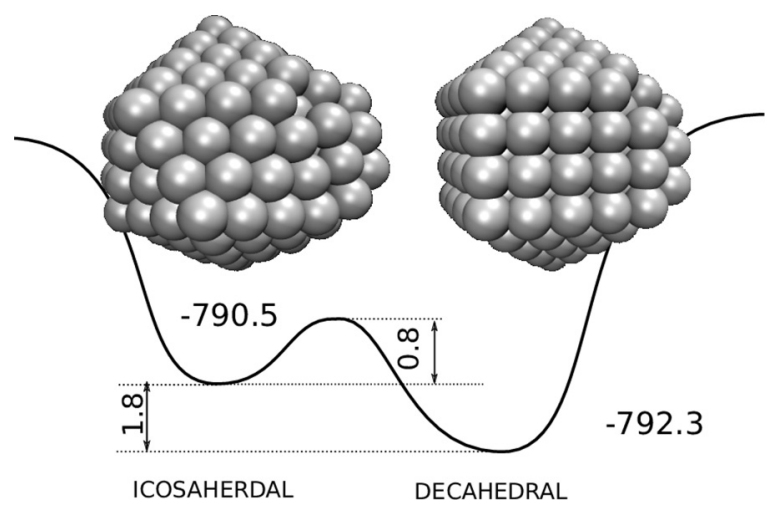

FIG. 3. The potential energy surface for the same 248 atom copper nanocluster as in Figs. 1 and 2. of clusters with nonperfect structure it is a very sophisticated problem to distinguish the contribution from the defects and distorted facets to the potential energy, however, a very good understanding of the trends could be gathered from models such as described in Ref. [28].

We applied the nudged elastic band (NEB) method to obtain the height of the potential energy barrier for the transition from icosahedral to decahedral morphology [Fig. 2(a)]. This allows us to plot a potential energy surface of this particular cooper nanocluster consisting of 248 atoms (Fig. 3). The icosahedral phase corresponds to a minimum with a potential energy of $-790.5 \mathrm{eV}$ and the decahedral phase corresponds to a deeper local minimum with a potential energy of $-792.3 \mathrm{eV}$. The barrier the cluster needs to overcome in order to minimize its energy is $0.8 \mathrm{eV}$. Besides, we calculated the barrier heights for different sizes of NCs. The cluster consisting of 185 atoms has a $0.6 \mathrm{eV}$ barrier and the 376-atom cluster has to overcome a barrier of $2 \mathrm{eV}$.

It is known, that icosahedron is the favorable morphology in many noble metals [14] at small sizes due to its small surface area [see Fig. 4(a)]. Thus icosahedron morphology minimizes the surface area, but causes relatively large internal stress. On the contrary, the decahedron morphology has a smaller internal stress but a larger surface area. If the impinging atoms during the growth are not able to diffuse far enough to form an uniform new layer, and thus instead form islands on the facets of the icosahedron, Fig. 4(b), then the surface area significantly increases. Thus, the surface area of the nonperfect icosahedron is large as well as its internal stress. A transition to the decahedral phase minimizes the internal stress though the surface area increases. Still, as one sees in Fig. 3, the potential energy of the decahedral phase is lower than that of the imperfect icosahedron.

Another way to minimize the energy is to redistribute the impinging atoms more uniformly, thus preventing islands from forming on the cluster surface and strong expansion of the surface area. Statistics of the simulated clusters (Table I) witnesses that the higher the temperature the lower the probability of the transition to the decahedral phase. This fact can be explained by the rate of diffusion, which is higher at high temperatures. The faster diffusion preserves the layer-by-layer growth. Moreover we have observed that small islands may diffuse, thus redistributing extra atoms more uniformly on the surface of the cluster and thereby decreasing the surface area. At the same time, it is clear that growth processes that restrict diffusionlike growth with normal incidence particles or the IGA process, where the diffusion is limited by the low energy of the impinging particle, have noticeably higher statistics for the morphology transitions. All these observations demonstrate how important the diffusion is in the morphology transition. But exactly how does a nonperfect icosahedron transform into a decahedron?

Detailed observations of hundreds of our NC growth simulations allowed us to identify a pattern and describe the mechanism of the morphology transitions from Ih to Dh phase. This mechanism has very simple foundations and thus seems very elegant. The basic idea is the following: the formation of islands on the surface disturbs the perfect facets of the icosahedron [Fig. 4(a)]. This disturbance can be localized in a certain area of the $\mathrm{NC}$, which could be 

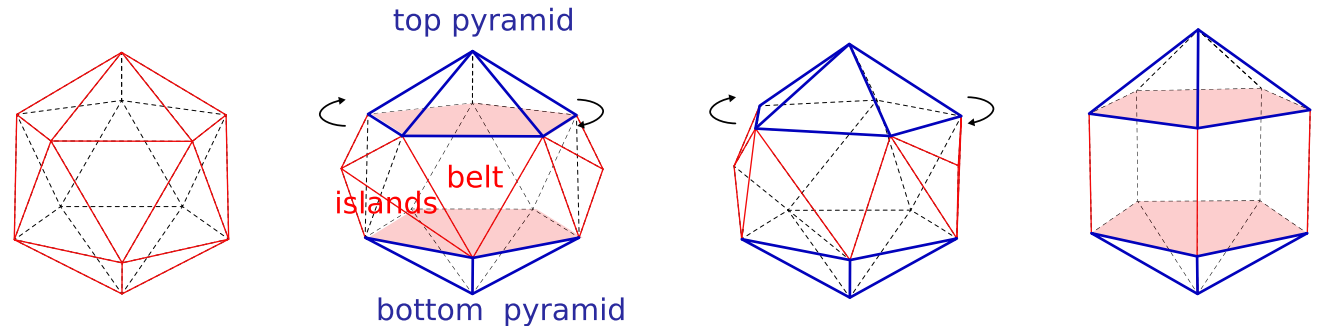

FIG. 4. (Color online) Mechanism of the morphology transitions from Ih to Dh phase: (a) a perfect icosahedron; (b) impinging atoms form islands on the surface of the cluster, thus increasing the surface energy; the most disturbed facets could be described as located in some kind of belt; (c) the bottom and top pentagonal pyramids twist with respect to each other and a fractional part of the islands on the facets incorporates into the cluster layers; (d) aligned edges of the new 100 facets and with all atoms in between the slices rearranged into a fcc structure.

considered as a "belt" formed by the extra atoms [Fig. 4(b)]. Above and under this belt one can distinguish two pentagonal pyramids with much less disorder. These pyramids do not require much rearrangement, because they should remain the same in the decahedral cluster. They only need to be twisted with respect to each other in order to align their edges and form the new rectangular facets of a decahedron, Figs. 4(c) and 4(d). During these relative rotations the atoms in the belt undergo major rearrangements. This includes the formation of fcc-coordinated atoms from hep ones and incorporation of the extra atoms from the surface into new layers. Let us underline that Fig. 4 is just a sketch of the transition process and therefore it represents a simplified case. However, in an arbitrary disordered cluster with the icosahedral structure due to its highly symmetrical geometry the part with the highest disorder should be considered as the belt, while the less perturbed ones should represent the upper and bottom pyramids.

Baletto et al. [17] suggested that Ih to Dh transition in silver nanoclusters goes through the melted phase of the cluster and that the new phase is formed from an amorphous structure. However, Mackay first described the diamondsquare mechanism of the direct geometrical transition from a cuboctahedron into an icosahedron [29]. This mechanism was later found in different systems $[12,15,19,30]$. However, the transition was only studied in perfect structures and the influence of the disorder on its mechanism was not considered. Thus the earlier works did not account for the influence of the kinetics, which is believed to be the morphology-determining process at realistic conditions. The mechanism suggested in the present Rapid Communication extends the diamond-square mechanism to the clusters with significant disorder and thus brings us closer to realistic growth conditions.

We have analyzed more than 700 simulations and we have shown that the mechanism of the morphology transition remains the same for all considered sizes, temperatures, and even energies of the impinging particles. Moreover, the present mechanism describes not only the perfect structure transitions but all the intermediate icosahedral clusters. This allows us to claim that this is a more universal mechanism of the solid-solid Ih-Dh morphology transition in $\mathrm{Cu}$ nanoclusters. Moreover, a transparent physical picture of the transition allows us to believe that it should be general, and therefore of importance for other systems.

In summary, we have reported the mechanism of solidsolid transition from $\mathrm{Ih}$ to $\mathrm{Dh}$ phase in $\mathrm{Cu}$ nanoclusters, and suggested an explanation of the cause of a morphology transition in Ih nanoclusters deep within their thermodynamic stability field. The origin of the transition has been associated with a formation of distorted NCs during the growth process with islands of incoming atoms localized in certain parts of the grown cluster. The distortions change the energy balance between Ih and Dh phases, leading to the changes of the morphology of the NCs. We revealed the role of diffusion on the morphology transition and showed how different facets of the cluster will transform during the transition. The fundamental understanding of the morphology transition mechanism allows us to suggest that varying the diffusion length in the growth process can be used to influence the cluster morphology. Considering the fact that the transition barrier increases with the increase of the cluster size, we expect that once the morphology is set at a certain large size it is very unlikely to change; a fact that again seems very convenient for tailoring the morphology of the grown nanoclusters.

We gratefully acknowledge useful discussions with Prof. Ulf Helmersson, Prof. Nils Brenning, and Dr. Iris Pilch. The work was financially supported by the Knut and Alice Wallenberg Foundation through Grant No. 2012.0083. I.A.A. is grateful for the support provided by the Swedish Foundation for Strategic Research (SSF) program SRL Grant No. 10-0026. The support by the grant from the Ministry of Education and Science of the Russian Federation (Grant No. 14.Y26.31.0005) is gratefully acknowledged. The calculations were performed on resources provided by the Swedish National Infrastructure for Computing (SNIC) at the National Supercomputer Center (NSC).
[1] F. Baletto and R. Ferrando, Rev. Mod. Phys. 77, 371 (2005).

[2] A. I. Ekimov, A. L. Efros, and A. A. Onushchenko, Solid State Commun. 56, 921 (1985).
[3] G. Prieto, J. Zecevic, H. Friedrich et al., Nat. Mater. 12, 34 (2013).

[4] B. R. Cuenya, Thin Solid Films 518, 3127 (2010). 
[5] C. R. Henry, Surf. Sci. Rep. 31, 235 (1998).

[6] J. A. Enterkin, K. R. Poeppelmeier, and L. D. Marks, Nano Lett. 11, 993 (2011).

[7] A. S. Urban, X. Shen, Y. Wang, N. Large, H. Wang, M. W. Knight, P. Nordlander, H. Chen, and N. J. Halas, Nano Lett. 13, 4399 (2013).

[8] M. A. Garcia, J. Phys. D: Appl. Phys. 44, 283001 (2011).

[9] T. A. Taton, C. A. Mirkin, and R. L. Letsinger, Science 289, 1757 (2000).

[10] Y. C. Chao, R. Jin, and C. A. Mirkin, Science 297, 1536 (2002).

[11] J. Uppenbrink and D. J. Wales, J. Chem. Soc. Faraday Trans. 87, 215 (1991).

[12] D. J. Wales and L. J. Munro, J. Phys. Chem. 100, 2053 (1996).

[13] L. D. Marks, Philos. Mag. A 49, 81 (1984).

[14] F. Baletto, R. Ferrando, A. Fortunelli, F. Montalenti, and C. Mottet, J. Chem. Phys. 116, 3856 (2002).

[15] K. Koga, T. Ikeshoji, and K. I. Sugawara, Phys. Rev. Lett. 92, 115507 (2004).

[16] Y. Feng, L. Hui, H. Wenlai, and Y. Jun, CrystEngComm 16, 8910 (2014).

[17] F. Baletto, C. Mottet, and R. Ferrando, Phys. Rev. Lett. 84, 5544 (2000).
[18] F. Baletto, C. Mottet, and R. Ferrando, Phys. Rev. B 63, 155408 (2001).

[19] Y. K. Lan, C. H. Su, W. H. Sun, and A. C. Su, RSC Adv. 4, 13768 (2014).

[20] I. Pilch, D. Söderström, N. Brenning, and U. Helmersson, Appl. Phys. Lett. 102, 033108 (2013).

[21] I. Pilch, D. Söderström, M. I. Hasan, U. Helmersson, and N. Brenning, Appl. Phys. Lett. 103, 193108 (2013).

[22] A. A. Tal, E. P. Münger, I. A. Abrikosov, N. Brenning, I. Pilch, and U. Helmersson, Phys. Rev. B 90, 165421 (2014).

[23] S. M. Foiles, M. I. Baskes, and M. S. Daw, Phys. Rev. B 33, 7983 (1986).

[24] S. Nosé, J. Chem. Phys. 81, 511 (1984).

[25] LAMMPS, http://lammps.sandia.gov.

[26] A. Stukowski, Modelling Simul. Mater. Sci. Eng. 20, 045021 (2012).

[27] G. J. Ackland and A. P. Jones, Phys. Rev.B 73, 054104 (2006).

[28] E. Ringe, B. Sharma, A.-I. Henry, L. D. Marks, and R. P. Van Duyne, Phys. Chem. Chem. Phys. 15, 4110 (2013).

[29] A. L. Mackay, Acta Crystallogr. 15, 916 (1962)

[30] G. Rollmann, M. E. Gruner, A. Huncht, R. Meyer, P. Entel, M. L. Tiago, and J. R. Chelikowsky, Phys. Rev. Lett. 99, 083402 (2007). 